\title{
Machine Learning for Next-generation Printed Technologies
}

\author{
Litty V. Thekkekara ${ }^{1,2 *}$, Shamini P. Baby ${ }^{4}$, Jeffery Chan ${ }^{4}$, Ivan Cole ${ }^{3}$ \\ 1. Functional Material and Microsystems Research Group and the Micro Nano Research Facility, RMIT University, GPO Box \\ 2476, Melbourne, Victoria 3001, Australia \\ 2. ARC Centre of Excellence for Transformative Meta-Optical Systems, RMIT University, GPO Box 2476, Melbourne, \\ Victoria 3001, Australia \\ 3. School of Engineering, RMIT University, GPO Box 2476, Melbourne, Victoria 3001, Australia \\ 4. School of Computer Science, RMIT University, GPO Box 2476, Melbourne, Victoria 3001, Australia
}

${ }^{\star}$ Corresponding Author: Litty V. Thekkekara, E-mail: littyvarghese.thekkekara@rmit.edu.au

\section{Abstract}

Modern science advances towards the development of lightweight wearable and portable applications for the promotion of human-machine interfaces. Among them, the most beneficial ones include the technologies for healthcare, telecommunications, and energy resources. Recent developments in the additive manufacturing otherwise 3D printing sector are promising for largescale applications. It promotes cost-effective production of technologies like sensors, lab on chips, solar cells, and energy storage. However, these applications' efficiency is lower to technologies fabricated using other methods like chemical approaches due to the non-optimized parameters involved in the fabrication and characterization phases. Machine learning on the other hand expands its science and engineering capabilities. It has a broader opportunity to support 3D printing to develop the potentials and efficiency through effective prediction methods for printing methods and design aspects. In this review, we discuss the use of machine learning prediction algorithms for technologies using 3D printing.

\section{Keywords}

Additive manufacturing; 3D printing; Printed technologies; Artificial intelligence; Machine learning; Data analysis

\section{INTRODUCTION}

Additive manufacturing (AM) ${ }^{[1]}$, also known as $3 \mathrm{D}$ printing, is used to create objects using layer-by-layer 
fabrication methods using a CAD design model. Rapid fabrication time and better process control for even arbitrary shape formation from nano to mesoscale results in cost-effective interactive devices with minimum material wastage and lower energy requirements, ensuring the promising future of the $3 \mathrm{D}$ printing industries ${ }^{[2]}$. Different 3D printing methods include binder jetting, material extrusion, material jetting, powder bed fusion, sheet lamination, direct energy deposition, metal casting, and photopolymerization methods like dynamic laser printing (DLP) and Stereolithography (SLA) ${ }^{[3]}$.

It profoundly impacts areas like automotive, lightweight wearables, portable electronics, energy storage, solar cells, optics, bioengineering, medical applications, and the fashion industry ${ }^{[4]}$. The applications are not limited to the previously listed, as discovery of materials that support the more dimensions for the fabrication, like $4 \mathrm{D}$ printing promotes new applications ${ }^{[5]}$.

However, the 3D printing process-based applications have several limitations in achieving the best performance due to the non-optimal final structures ${ }^{[6]}$. The primary issue preventing the development of an ideal output structure using 3D printing processing could be the reduced heat dissipation time in the fabrication materials. It can lead to difficulties in developing desired shapes in the output with non-desired roughness. Besides, the stitching errors contributed by the $3 \mathrm{D}$ printing source can result in resolution issues, which results in misconfigured structures, voids, and interlayer spacing problems in the final output.

Several other factors, like the mechanical delays in the printing system, non-coordination between computer software used for the control and the printing system, thermal relaxation of dynamic optical and other mechanical systems, non-optimization of the designs utilized, can influence the printing process ${ }^{[7]}$. In recent years, the utilization of artificial intelligence (AI) and its sub-fields like machine learning (ML) for 3D printing show a promise in developing a self-intelligent automated fabrication process through assistance in design, choosing material, material tuning, process optimization, in situ monitoring, defect recognition, cloud service, and cybersecurity ${ }^{[8]}$.

With the utilization of the processed information and data, the ML training network figure out how to make decisions ${ }^{[9]}$. ML is dynamic, implying that it can alter itself when exposed to more information. The 'learning' part of ML means that the algorithms attempt to limit the errors and boost likelihood of their predictions being valid.

The field of ML has organized around three primary research: (1) task-oriented studies, involving the development and analysis of learning systems to improve performance in a predetermined set of tasks, (2) cognitive simulation used for investigation and computer simulation of human learning processes and finally, (3) analysis such as the theoretical space exploration of possible learning methods and algorithms independent of application domain ${ }^{[10]}$.

Deep Learning (DL) which is a subset of ML incorporates computational models and algorithms that imitate the architecture of the brain's biological neural networks, which are termed artificial neural networks $(A N N s)^{[11]}$. Deep Neural Networks (DNNs) are used in various applications, such as object recognition in images and acoustic processing for speech recognition ${ }^{[12]}$. Whenever the brain gets new data, it attempts to contrast with known data to make sense. The brain decodes the information through labeling and assigning the items to various categories, and DL employs the same concept.

'Deep' is a technical term and refers to the number of layers in an ANN. There are three types of layers: (a) an input layer (receives the input data), (b) a output layer (produces the result of data processing), and (3) the 
hidden layer (extracts the patterns within the data) ${ }^{[13]}$. While the information moves from one hidden layer to another, more superficial features recombine and recompose complex elements. DL works exceptionally well on unstructured data and has higher accuracy than traditional ML approaches for unsupervised training, but requires a considerable volume of training data, along with expensive hardware and software.

Here, we discuss the current use of ML in 3D printing, and perspectives about the improvement in this area using advanced ML methodologies significantly to optimize the prefabrication design process, defect/failure detection, real-time 3D printing control/failure compensation, predictive maintenance, cost optimization and photopolymerization using ML-based algorithm to maximize control on chemicals and energy dose input.

\section{ML FOR 3D PRINTING APPLICATIONS}

There has been a lot of recent interest in adopting ML methods for scientific and engineering applications using 3D printing ${ }^{[14]}$. Recently, a lot of attention has been given for printable graphical codes enabling a link between the physical and digital worlds, which is of great interest for anti-counterfeiting, Internet of Things (IoT), and brand protection applications ${ }^{[15]}$.

In a demonstrated work, an automated ML segmentation procedure to create a virtual object to be printed ${ }^{[16]}$. They made an accurate 3D printed core sample replicas using an ML image processing tool (MLIPT). Another application of ML technique $\mathrm{s}$ in the prediction of the hole-filling in pin-in-paste technology ${ }^{[17]}$. A detailed evaluation of ML-based prediction methods is performed in this research, including artificial neural networks (ANN), adaptive neuro-fuzzy inference systems (AFNIS), and gradient boosted decision trees to optimize the process parameters of pin-in-paste. Another relevant 3D printing application that has potential use is concrete printing. In this application, the ANN model optimizes the surface finish quality and potentially affects bonding strength between layers ${ }^{[18]}$.

In addition to all these printable applications, shape accuracy is an important performance measure or product built via 3D printing. Some works ${ }^{[19]}$ adopted Gaussian process regression to capture spatial correlations. The printed 2-D and 3-D shapes are used to demonstrate the proposed modeling framework and derive new process insights for fabrication process. The proposal for developing a useful tool with the potential for broad application in planning and optimizing 3D printing of soft materials with Hierarchical ML algorithms $(\mathrm{HML})^{[20]}$. HML algorithm predicts the problematic physical system behaviours using sparse data sets through the integration of physical modeling using statistical learning. This methodology simultaneously optimizes material, formulation, and process variables.

In a report on manufacturing a 3D printer, the functional surfaces of printing medium are developed with DL's help ${ }^{[21]}$. This methodology is beneficial for printing highly biological samples like bone prosthetics, regenerative biomedicines, and fabrication directly on the human body's internal organs like implantation of sensors for in-situ monitoring of lungs' functioning and heart. 3D printing of biomaterials for tissue-engineering by utilizing random forest ML algorithms is a significant step towards more accurate and efficient biomedical applications ${ }^{[22]} 3 \mathrm{D}$ printed personalized pharmaceuticals and medicines depending on the individual patient necessities are now accomplished using ML algorithms ${ }^{[23]}$.

ML applications in optical technologies attain exceptional attention in recent years. The ML use gained rapid growth in the sectors like laser beam-material interactions for fundamental material science studies helped understand the physical and chemical property changes ${ }^{[24]}$. ML is used to enhance the disc's data storage capacity by fabricating the optical structures beyond the diffraction limit of light ${ }^{[25]}$. ML algorithms have helped 
to develop and discover better photonic designs for optical communications, augmented realities, displays, holography, meta-optics, adaptive optics, metrology, and quantum optics using 3D printing ${ }^{[26]}$. Apart from these studies, recently, researchers are successful in 3D printing a framework of multiple layers of diffractive surfaces, which in the collective form an optical analog of a neural network for statistical learning and termed as a diffractive deep neural network $\left(\mathrm{D}^{2} \mathrm{NN}\right)^{[26]}$. In the framework, both the network's interference and prediction mechanism are all-optical through a computer-controlled design.

We have summarized the ML applications in different steps of developing a 3D printing from material selection towards the optimization and characterization of the processed device in Figure 1.

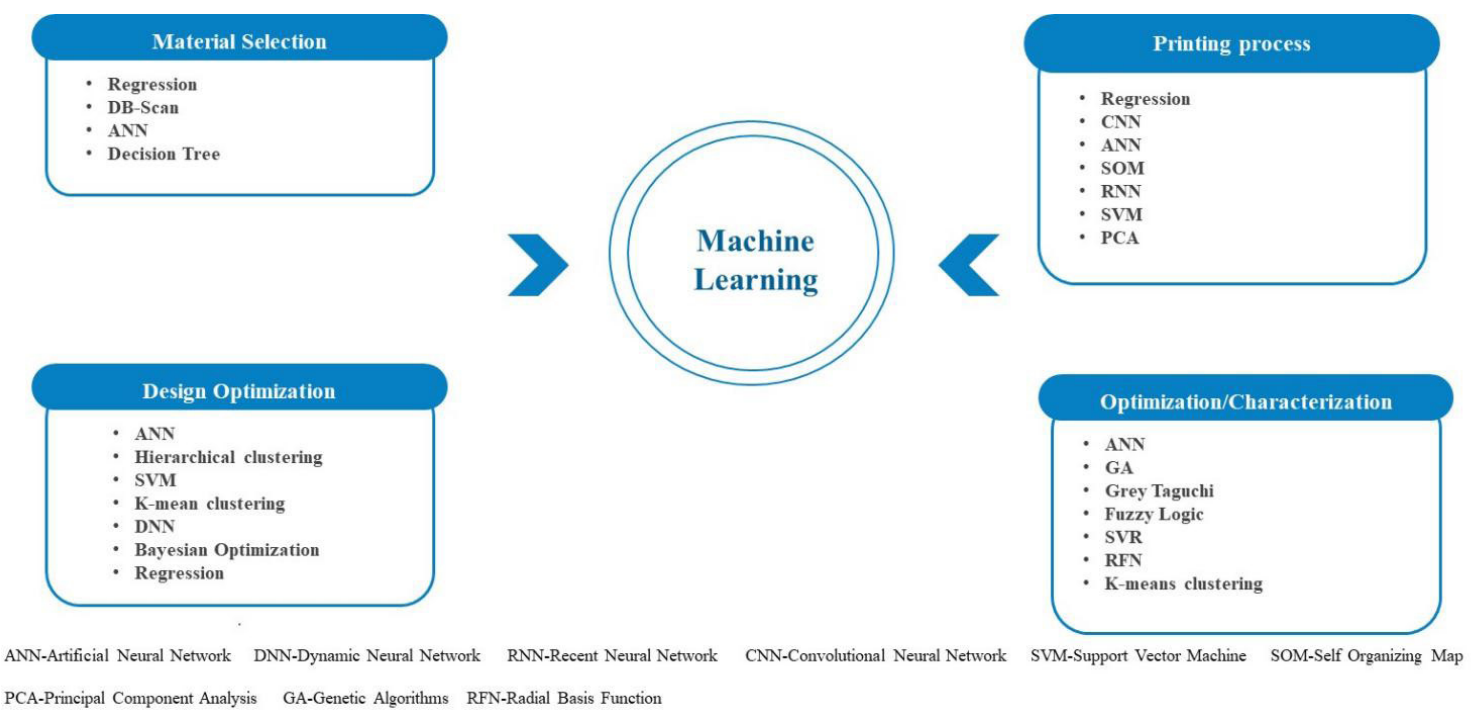

Figure 1. ML applications in the development of 3D printing technology.

\section{CHALLENGES OF ML USE IN 3D PRINTING}

For ML, the training data is the critical input where having the right quality and quantity of data sets is essential to get accurate results. The larger the ML algorithm's training data, it will more likely to help the model to see diverse types of objects, making it easier to recognize and generalize to diverse real-life scenarios. Data collection is a significant bottleneck in ML. We envision that requirement for (training) data play a larger role in specifying ML systems than for conventional methods. This information needs to be elicited from the problem domain and serves as an input ${ }^{[27]}$.

It is known that the major time for running ML end-to-end is spent on preparing the data, which includes collecting, cleaning, analyzing, visualizing, and feature engineering. There are two reasons for which data collection has recently become a critical issue. First, as ML is becoming more widely used, we can see new applications that do not necessarily have enough labeled data. Second, unlike traditional ML, deep learning techniques can be used by unsupervised training to generate automatic features, which saves feature engineering costs, but in return, may require more massive amounts of labeled data ${ }^{[27]}$.

Supervised ML models are successfully used to respond to a whole range of challenges. However, these models need more data, and their performance relies heavily on the size of training sets available. In many cases, it is not easy to create training datasets that are large enough, particularly for engineering tasks. It is impossible to precisely estimate the minimum amount of data required for AI projects. The nature of every project will significantly influence the amount of data we will need. Many 3D printing systems do record data 
during the builds, and that this data could be curated and collected to assist with ML. Besides, other factors such as 'number of categories to be predicted' and 'model performance' should be considered to make an accurate estimate.

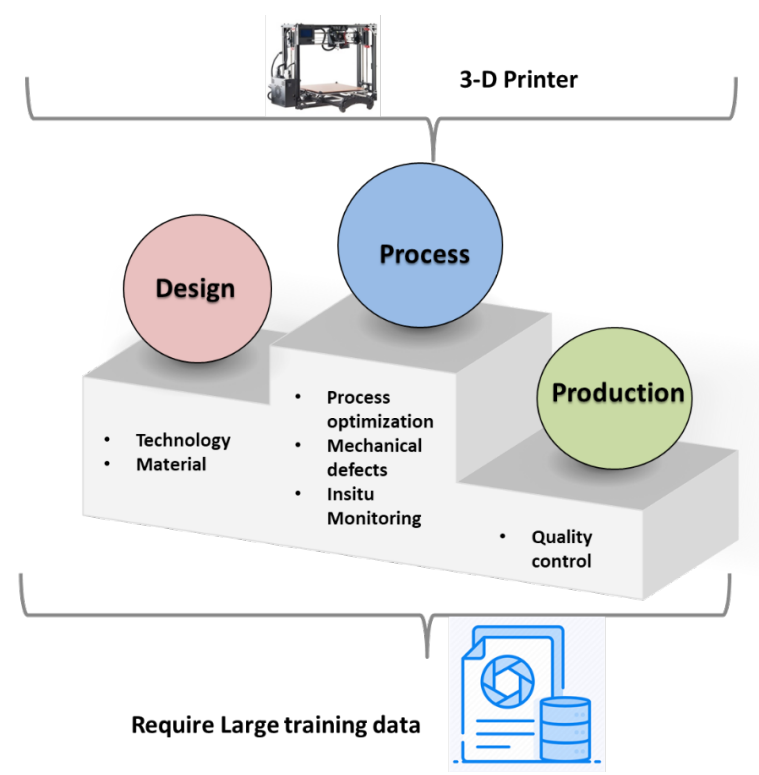

Figure 2. Challenges in the implementation of ML algorithms in printing technologies.

\section{PERSPECTIVES}

There is a wide range of ML algorithms available, and their applications are immense. Here we can adopt a suitable method that gives minimum errors and maximum accuracy for our printing applications. It will enable the analysis to focus on the available scientific measurements and the actual optimization process using the essential experimental and simulated data points. One successful example in this field is a study conducted by Google Health to measure breast cancer signs with few input variables ${ }^{[28]}$. Another approach is in the designing of pharmacologically relevant chemical space with drug-like molecular entities on demand with limited training data ${ }^{[29]}$. The final model aims to have better accuracy and a lesser time frame than numerical simulations.

Fault management is a functional area of systems management related to the detection, prediction, isolation, and prevention of faults. A model is trained by looking at the system's fault-free state, and can be used in all printing processes today as they have much simpler requirements to system expertise or data needed or training the fault-free models ${ }^{[30]}$. AI can have a more significant influence in the field of automating $3 \mathrm{D}$ printing workflows. The printability of an object can be analyzed before starting the fabrication process. The quality of a part can also be predicted, and the process can be controlled to avoid printing errors, effectively saving time. Material selection can also be automated with AI depending on the requirements of the design to be printed. A flowchart for the process is as shown in Figure 3 below.

We propose a model that can predict accurate results with the supply of minimum input data for printable scientific applications using appropriate design and suitable materials. Using the data prediction aspects of machine learning by incorporating computer simulation, develop an ML model that can predict better and accurate results using lesser input data. The final model aims to have better accuracy and a more secondary time frame than numerical simulations. Design optimization of printable applications can be done with the help of a Deep Neural network (DNN), which is a self-learning model and accurate by nature. In this approach, performance improvement and optimization methodologies such as Backtracking in $\mathrm{DNN}^{[31]}$ and Bayesian 
optimization ${ }^{[32]}$ can be applied iteratively to get precise outcomes. At each iteration, local optima can be evolved, and that can be used as the input to the next iteration. In this way, the performance will increase and minimize errors, which will result in an optimized design model. A perspective for our hypothesis is given in Figure 4 below.

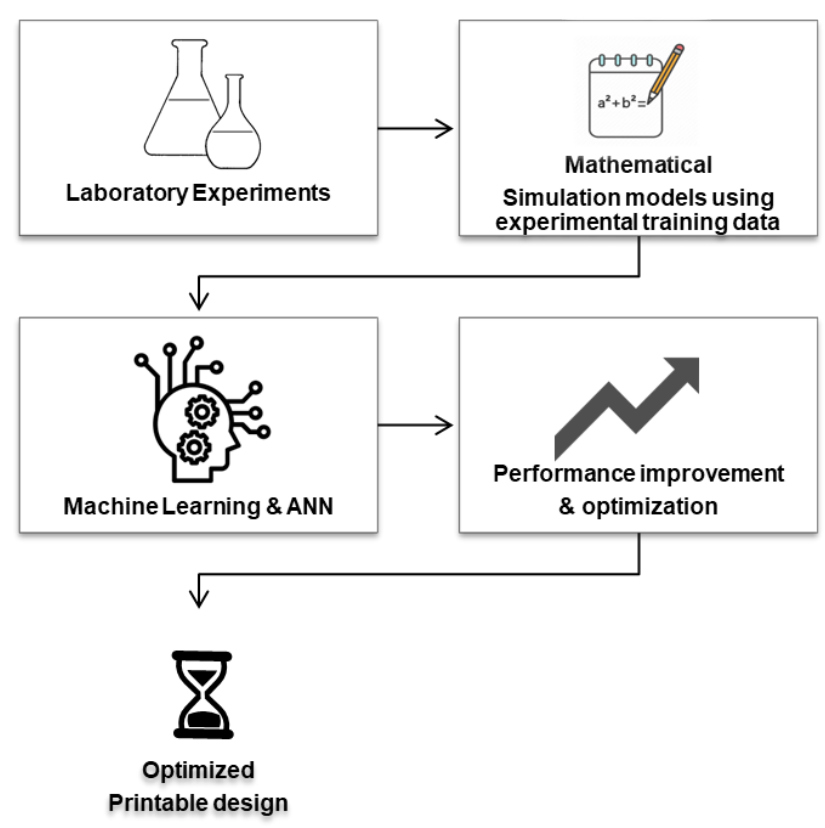

Figure 3. Design optimization process.

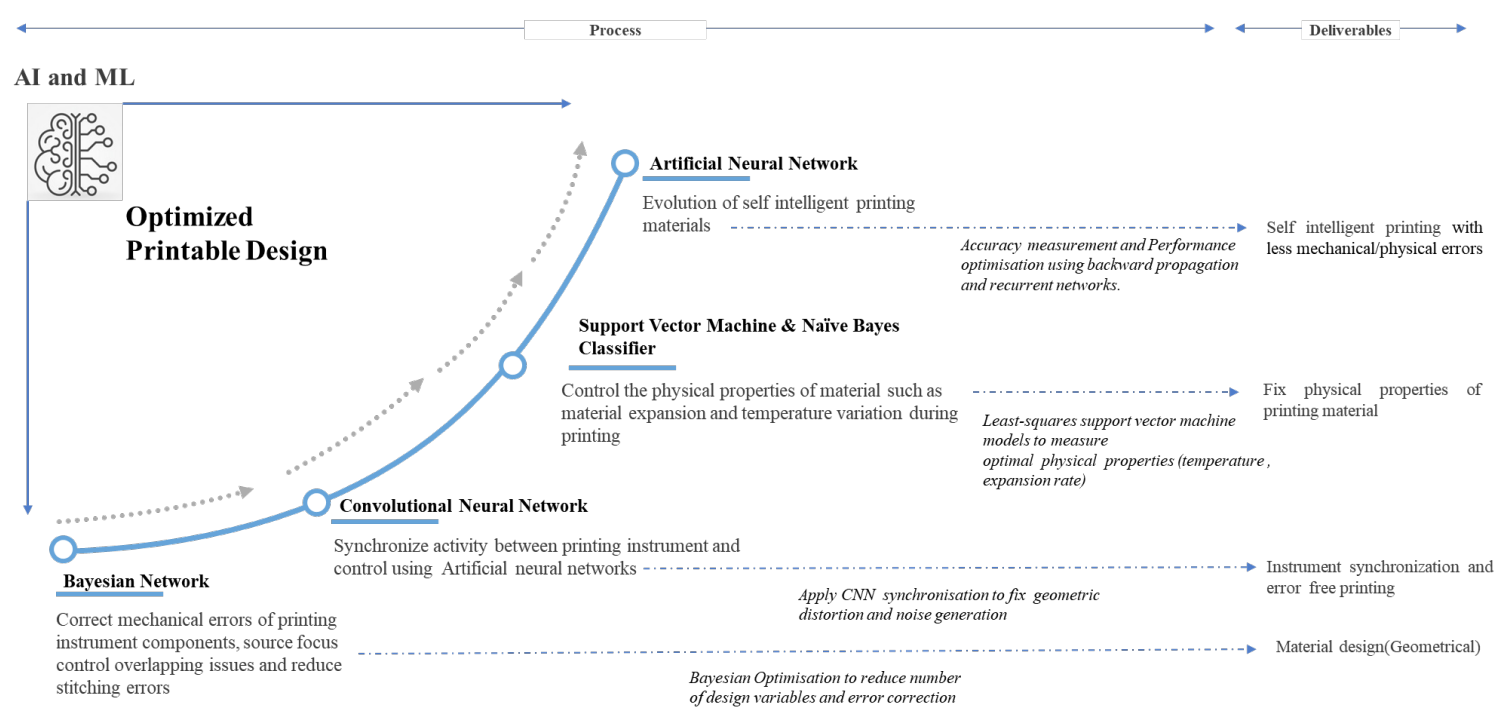

Figure 4. Perspective for ML assisted printing technologies.

In conclusion, we propose a model that can predict accurate results with minimum input data supply. We consider printable scientific applications using appropriate design, suitable materials, and optimization of the fabrication process. This research aims to utilize the data prediction aspects of ML by incorporating computer simulation techniques. In this process, we can develop an ML model that can predict better and accurate results using lesser input data for the feed loop mechanism. 


\section{ACKNOWLEDGMENTS}

This research was supported (partially or fully) by the Australian Government through the Australian Research Council's Discovery Projects funding scheme (project DP170103174).

\section{AUTHOR CONTRIBUTIONS}

All authors contributed equally to the design, writing, and editing of the manuscript.

\section{COMPETING INTERESTS}

The authors declare no competing financial interests.

\section{REFERENCE}

[1] I. Gibson, D. W. Rosen, B. Stucker, Additive manufacturing technologies, Springer, 2014.

[2] a) H. Lipson, M. Kurman, Fabricated: The new world of $3 D$ printing, John Wiley \& Sons, 2013; b) B. Berman, Business horizons 2012, 55, 155; c) L. Jonušauskas, D. Gailevičius, S. Rekštytè, T. Baldacchini, S. Juodkazis, M. Malinauskas, Optics express 2019, 27, 15205.

[3] a) T. D. Ngo, A. Kashani, G. Imbalzano, K. T. Nguyen, D. Hui, Composites Part B: Engineering 2018, 143, 172; b) S. Maruo, O. Nakamura, S. Kawata, Optics letters 1997, 22, 132; c) S. Kawata, H.-B. Sun, T. Tanaka, K. Takada, Nature 2001, 412, 697.

[4] a) J. Chang, T. Ge, E. Sanchez-Sinencio, presented at 2012 IEEE 55th International Midwest Symposium on Circuits and Systems (MWSCAS) 2012; b) A. Vanderploeg, S.-E. Lee, M. Mamp, International Journal of Fashion Design, Technology and Education 2017, 10, 170; c) S. Khan, L. Lorenzelli, R. S. Dahiya, IEEE Sensors Journal 2014, 15, 3164; d) Q. Yan, H. Dong, J. Su, J. Han, B. Song, Q. Wei, Y. Shi, Engineering 2018, 4, 729; e) C. Schubert, M. C. Van Langeveld, L. A. Donoso, British Journal of Ophthalmology 2014, 98, 159; f) J. A. Lewis, B. Y. Ahn, Nature 2015, 518, 42; g) S.-Y. Wu, C. Yang, W. Hsu, L. Lin, Microsystems \& Nanoengineering 2015, 1, 1; h) Y. L. Kong, I. A. Tamargo, H. Kim, B. N. Johnson, M. K. Gupta, T.-W. Koh, H.-A. Chin, D. A. Steingart, B. P. Rand, M. C. McAlpine, Nano letters 2014, 14, 7017; i) G. Comina, A. Suska, D. Filippini, Lab on a Chip 2014, 14, 424; j) L. V. Thekkekara, M. Gu, Scientific reports 2017, 7, 45585; k) Y. Liu, T. T. Larsen-Olsen, X. Zhao, B. Andreasen, R. R. Søndergaard, M. Helgesen, K. Norrman, M. Jørgensen, F. C. Krebs, X. Zhan, Solar energy materials and solar cells 2013, 112, 157; 1) A. Ghilan, A. P. Chiriac, L. E. Nita, A. G. Rusu, I. Neamtu, V. M. Chiriac, Journal of Polymers and the Environment 2020, 1.

[5] a) A. Mitchell, U. Lafont, M. Hołyńska, C. Semprimoschnig, Additive Manufacturing 2018, 24, 606; b) D. Bourell, J. P. Kruth, M. Leu, G. Levy, D. Rosen, A. M. Beese, A. Clare, CIRP Annals 2017, 66, 659.

[6] a) B. Ahuja, M. Karg, M. Schmidt, presented at Laser 3d manufacturing II 2015; b) S. Yang, Y. F. Zhao, The International Journal of Advanced Manufacturing Technology 2015, 80, 327; c) N. Kouraytem, X. Li, W. Tan, B. Kappes, A. Spear, Journal of Physics: Materials 2020.

[7] a) D. I. Wimpenny, P. M. Pandey, L. J. Kumar, Advances in 3D Printing and Additive Manufacturing Technologies, Springer Singapore Pte. Limited, Singapore, SINGAPORE 2016; b) G. A. Adam, D. Zimmer, Rapid Prototyping Journal 2015.

[8] a) U. Delli, S. Chang, Procedia Manufacturing 2018, 26, 865; b) Z. Jin, Z. Zhang, J. Ott, G. X. Gu, Additive Manufacturing 2020, 101696; c) H. Zhang, S. K. Moon, T. H. Ngo, ACS applied materials \& interfaces 2019, 11, 17994; d) T. Wang, T.-H. Kwok, C. Zhou, S. Vader, Journal of manufacturing systems 2018, 47, 83; e) T. DebRoy, T. Mukherjee, H. Wei, J. Elmer, J. Milewski, Nature Reviews Materials 2020, 1; f) C. Wang, X. P. Tan, S. B. Tor, C. S. Lim, Additive Manufacturing 2020, 36, 101538; g) G. D. Goh, S. L. Sing, W. Y. Yeong, Artificial Intelligence Review 2020, 1; h) L. Scime, J. Beuth, Additive Manufacturing 2019, 25, 151; i) L. Scime, J. Beuth, Additive Manufacturing 2018, 19, 114; j) A. Caggiano, J. Zhang, V. Alfieri, F. Caiazzo, R. 
Gao, R. Teti, CIRP Annals 2019, 68, 451.

[9] F. Pesapane, M. Codari, F. Sardanelli, European radiology experimental 2018, $2,35$.

[10] R. MICHALSIK, J. Carbonell, L. MICHE, Palo Alto: Tioga Publishing, 1983.

[11] J. Schmidhuber, Neural networks 2015, 61, 85.

[12] M. E. Morocho-Cayamcela, H. Lee, W. Lim, IEEE Access 2019, 7, 137184.

[13] B. F. King Jr, Am Roentgen Ray Soc, 2017.

[14] D. Jakhar, I. Kaur, Clinical and experimental dermatology 2020, 45, 131.

[15] O. Taran, S. Bonev, S. Voloshynovskiy, presented at ICASSP 2019-2019 IEEE International Conference on Acoustics, Speech and Signal Processing (ICASSP) 2019.

[16] A. Almetwally, H. Jabbari, Journal of Natural Gas Science and Engineering 2020, 76, 103192.

[17] P. Martinek, O. Krammer, Computers \& Industrial Engineering 2019, 136, 187.

[18] W. Lao, M. Li, T. N. Wong, M. J. Tan, T. Tjahjowidodo, Virtual and Physical Prototyping 2020, $15,178$.

[19] Q. Huang, Y. Wang, M. Lyu, W. Lin, IEEE Transactions on Automation Science and Engineering 2020.

[20] A. Menon, B. Póczos, A. W. Feinberg, N. R. Washburn, 3D Printing and Additive Manufacturing 2019, 6, 181.

[21] Z. Zhu, D. W. H. Ng, H. S. Park, M. C. McAlpine, Nature Reviews Materials 2021, 6, 27.

[22] A. Conev, E. E. Litsa, M. R. Perez, M. Diba, A. G. Mikos, L. E. Kavraki, Tissue Engineering Part A 2020.

[23] M. Elbadawi, B. Muñiz Castro, F. K. H. Gavins, J. J. Ong, S. Gaisford, G. Pérez, A. W. Basit, P. Cabalar, A. Goyanes, International Journal of Pharmaceutics 2020, 590, 119837.

[24] a) G. Casalino, Optics \& Laser Technology 2018, 100, 165; b) J. Zhou, B. Huang, Z. Yan, J.-C. G. Bünzli, Light: Science \& Applications 2019, 8, 1.

[25] P. R. Wiecha, A. Lecestre, N. Mallet, G. Larrieu, Nature nanotechnology 2019, 14, 237.

[26] a) J. Biamonte, P. Wittek, N. Pancotti, P. Rebentrost, N. Wiebe, S. Lloyd, Nature 2017, 549, 195; b) F. N. Khan, Q. Fan, C. Lu, A. P. T. Lau, Journal of Lightwave Technology 2019, 37, 493; c) F. N. Khan, C. Lu, A. P. T. Lau, presented at 2018 Optical Fiber Communications Conference and Exposition (OFC) 2018; d) Z. A. Kudyshev, A. V. Kildishev, V. M. Shalaev, A. Boltasseva, Nanophotonics 2020, 1; e) L. Li, H. Ruan, C. Liu, Y. Li, Y. Shuang, A. Alù, C.-W. Qiu, T. J. Cui, Nature communications 2019, 10, 1; f) X. Lin, Y. Rivenson, N. T. Yardimci, M. Veli, Y. Luo, M. Jarrahi, A. Ozcan, Science 2018, 361, 1004; g) H. Ren, W. Shao, Y. Li, F. Salim, M. Gu, Science Advances 2020, 6, eaaz4261; h) S. You, J. Guan, J. Alido, H. H. Hwang, R. Yu, L. Kwe, H. Su, S. Chen, Journal of Manufacturing Science and Engineering 2020, 142.

[27] J. P. Winkler, J. Grönberg, A. Vogelsang, presented at 2019 IEEE 27th International Requirements Engineering Conference (RE) 2019.

[28] S. M. McKinney, M. Sieniek, V. Godbole, J. Godwin, N. Antropova, H. Ashrafian, T. Back, M. Chesus, G. C. Corrado, A. Darzi, Nature 2020, 577, 89.

[29] M. Moret, L. Friedrich, F. Grisoni, D. Merk, G. Schneider, Nature Machine Intelligence 2020, $2,171$.

[30] F. Baumann, D. Roller, presented at MATEC web of conferences 2016.

[31] G. Chartrand, P. M. Cheng, E. Vorontsov, M. Drozdzal, S. Turcotte, C. J. Pal, S. Kadoury, A. Tang, Radiographics 2017, 37, 2113.

[32] F. Archetti, A. Candelieri, Bayesian Optimization and Data Science, Springer, 2019. 